\title{
Use of Potentially Nephrotoxic Medications by U.S. Adults with Chronic Kidney Disease: NHANES, 201 1-2016
}

\author{
Shaheen Kurani, ScM ${ }^{7}$, Molly Moore Jeffery, $P h D^{2}$, Bjorg Thorsteinsdottir, MD ${ }^{3,4}$, \\ LaTonya J. Hickson, MD ${ }^{3,5}$, Erin F. Barreto, PharmD, MSc ${ }^{3,6}$, Jordan Haag, PharmD', \\ Rachel Giblon, MS $S^{3}$, Nilay D. Shah, PhD ${ }^{2,3}$, and Rozalina G. McCoy, MD, $\mathrm{MS}^{2,3,4}$ (D)
}

\begin{abstract}
'Mayo Clinic Graduate School of Biomedical Sciences, Mayo Clinic, Rochester, MN, USA; ${ }^{2}$ Division of Health Care Policy \& Research, Department of Health Sciences Research, Mayo Clinic, Rochester, MN, USA; ${ }^{3}$ Mayo Clinic Robert D. and Patricia E. Kern Center for the Science of Health Care Delivery, Mayo Clinic, Rochester, MN, USA; ${ }^{4}$ Division of Community Internal Medicine, Department of Medicine, Mayo Clinic, Rochester, MN, USA; ${ }^{5}$ Division of Nephrology and Hypertension, Department of Medicine, Mayo Clinic, Rochester, MN, USA; ${ }^{6}$ Department of Pharmacy, Mayo Clinic, Rochester, MN, USA.
\end{abstract}

BACKGROUND: People with chronic kidney disease (CKD) are at risk for adverse events and/or CKD progression with use of renally eliminated or nephrotoxic medications.

OBJECTIVE: To examine the prevalence of potentially inappropriate medication (PIM) use by U.S. adults by CKD stage and self-reported CKD awareness.

DESIGN: Cross-sectional analysis of National Health and Nutrition Examination Surveys, 2011-2016

PARTICIPANTS: Non-pregnant adults with stages 3a (eGFR 45-59 mL/min/1.73 $\mathrm{m}^{2}$ ), 3b (eGFR 30-44), or 45 (eGFR < 30) CKD, stratified as CKD-aware/unaware.

MAIN MEASURES: PIMs were identified on the basis of KDIGO guidelines, label information, and literature review. We calculated proportions using any and individual PIMs, assessing for differences over CKD awareness within each CKD stage. Analyses were adjusted for age, sex, race/ethnicity, education, comorbidities, and insurance type.

KEY RESULTS: Adjusted proportions of U.S. adults taking any PIM(s) exceeded 50\% for all CKD stages and awareness categories, and were highest among CKDunaware patients with stages 4-5 CKD: 66.6\% (95\% CI, 55.5-77.8). Proton pump inhibitors, opioids, metformin, sulfonylureas, and non-steroidal anti-inflammatory drugs (NSAIDs) were all used frequently across CKD stages. NSAIDs were used less frequently when CKDaware by patients with stage 3a CKD $(2.2 \%$ [95\% CI, 0.3 to 4.7 ] vs. $10.7 \%$ [95\% CI, 7.6 to 13.8 ]) and stages $4-5$ CKD $(0.8 \%$ [95\% CI, -0.9 to 2.5 ] vs. $16.5 \%$ [95\% CI, 4.0 to 29.0]). Metformin was used less frequently when CKDaware by patients with stage 3b CKD $(8.1 \%$ [95\% CI, 0.3-15.9] vs. 26.5\% [95\% CI, 17.4-35.7]) and stages 45 CKD (none vs. 20.8\% [95\% CI, 1.8-39.8]). The impact of CKD awareness was statistically significant after correction for multiple comparisons only for NSAIDs in stage 3a CKD.

Electronic supplementary material The online version of this article (https://doi.org/10.1007/s11606-019-05557-8) contains supplementary material, which is available to authorized users.

Received October 22, 2019

Revised October 22, 2019

Accepted November 11, 2019

Published online December 2, 2019
CONCLUSIONS: PIMs are frequently used by people with CKD, with some impact of CKD awareness on NSAID and metformin use. This may lead to adverse outcomes or hasten $\mathrm{CKD}$ progression, reinforcing the need for improved medication management among people with $\mathrm{CKD}$.

J Gen Intern Med 35(4):1092-101

DOI: $10.1007 / \mathrm{s} 11606-019-05557-8$

(C) Society of General Internal Medicine 2019

\section{INTRODUCTION}

Thirty million U.S. adults - nearly $15 \%$ of the population - have some degree of kidney dysfunction [1], though many are unaware of their kidney impairment [2-5]. The contribution of chronic kidney disease (CKD) to the global health burden is expected to rise in coming years [6]. People with CKD commonly have multiple comorbidities and require many medications [7-9]. Medication use in the context of CKD is complicated, due to difficult risk/benefit decisions and altered drug pharmacokinetics and pharmacodynamics [10]. As a result, people with CKD are at risk for adverse events and/or CKD progression with use of renally eliminated or nephrotoxic medications [1114]. With the significant increase in polypharmacy over the last decade [15], it is increasingly important to understand potentially inappropriate medication (PIM) use by patients with CKD. Furthermore, efforts to reduce PIM use need to consider the potential impact of historically poor CKD awareness [3-5]; yet, whether and how individuals' awareness of their kidney dysfunction affects their PIM use remains unknown.

The KDIGO Clinical Practice Guideline for the Evaluation and Management of CKD strongly advises caution with use of potentially nephrotoxic and renally eliminated medications by people with eGFR $<60 \mathrm{~mL} / \mathrm{min} / 1.73 \mathrm{~m}^{2}$ [12]. While some of these medications are often clinically necessary for patients with CKD (e.g., diuretics), others are discouraged due to risk of harm and/or potential availability of safer treatment alternatives. These include non-steroidal anti-inflammatory drugs (NSAIDs), opioids, metformin, sulfonylureas, non-warfarin anticoagulants, bisphosphonates, proton pump inhibitors (PPIs), 
anti-gout medications, and select seizure medications [12]. These medications are either directly nephrotoxic or renally eliminated, and their inappropriate dosing or monitoring could confer substantial preventable harm among people with CKD [16]. Better understanding of the prevalence and factors associated with PIM use, as well as the impact of CKD awareness on PIM use, is necessary to develop targeted interventions that improve medication safety for people with CKD. We address this critical knowledge gap in the general population of noninstitutionalized U.S. adults using National Health and Nutrition Examination Survey (NHANES) data between 2011 and 2016.

\section{METHODS}

\section{Study Design}

We analyzed NHANES data for 2011-2016. NHANES uses stratified, multistage, probability-cluster sampling techniques to develop estimates representative of U.S. noninstitutionalized civilians. Data are collected from household interviews and standardized medical examinations. Blood samples are collected in mobile examination centers (MECs) and analyzed by NHANES staff. This study was exempt from review by Mayo Clinic Institutional Review Board as it uses de-identified data.

\section{Study Population}

We included adults ( $\geq 20$ years) who had data to estimate the glomerular filtration rate (eGFR) using the Chronic Kidney Disease Epidemiology Collaboration (CKD-EPI) equation [17]. We defined CKD as eGFR $<60 \mathrm{~mL} / \mathrm{min} / 1.73 \mathrm{~m}^{2}$, further categorized as stage $3 a$ (eGFR 45-59), 3 b (eGFR 30-44), or 4-5 (eGFR < 30). Measurement of serum creatinine was performed using the Beckman UniCel® DxC800 Synchron by Collaborative Laboratory Services, LLC after collection by NHANES MECs.

\section{Outcomes}

Primary outcome was report of any PIM use during the NHANES interview, confirmed by interviewer review of all medication containers used in the prior month. We identified PIMs based on the KDIGO Clinical Practice Guidelines [12], medication labels and Micromedex prescribing guidelines [18], and published literature [16, 19-21]. Included PIMs, detailed in eTable 1, were the following: NSAIDs, opioids, sulfonylureas, metformin, anticoagulants (direct oral anticoagulants and low molecular weight heparins), proton pump inhibitors (PPIs), bisphosphonates, gout medications, seizure medications, and miscellaneous others (amantadine, atenolol, quinidine, quinine, digoxin, lithium, tacrolimus, fenofibrate, acarbose, fexofenadine, and fesoterodine). Only systemic (not ophthalmic or topical) formulations were included. We secondarily specifically examined use of NSAIDs, opioids, sulfonylureas, metformin, and PPIs.

\section{Independent Variables}

CKD awareness was assessed by the response to "Have you ever been told by a doctor or other health professional that you had weak or failing kidneys? Do not include kidney stones, bladder infections, or incontinence." This approach is consistent with prior studies examining CKD awareness [2-4] and is used by the Centers for Disease Control and Prevention [5]. We included selected results for those without CKD (eGFR $\geq$ $60)$ to facilitate comparison with the broader noninstitutionalized U.S. adult population.

Interview responses were used to ascertain participants' age, sex, race/ethnicity, education level, and insurance type. Education was categorized as high school (HS) or less vs. more than HS (including unknown/refused). Insurance type was categorized as private, Medicare, Medicaid/dual eligible, other public health plan (SCHIP, military, Indian Health Service, state-sponsored, other governments), or uninsured (including unknown/missing insurance).

Comorbidities were ascertained from self-report, including diabetes, arthritis, hypertension, heart failure, and a cardiovascular disease (CVD) composite of coronary heart disease, angina, heart attack, and/or stroke. Participants with missing/"don't know" responses were classified as not having that comorbidity.

\section{Statistical Analysis}

We estimated the unadjusted proportion of NHANES participants taking one or more PIMs by CKD stage using NHANES-provided sampling design variables across all years of the study. Consequently, data estimates represent average values for the non-institutionalized U.S. adult population across the entire study period. Following NHANES recommended methodology [22], 95\% CIs for all estimates were constructed using the Taylor series (linearization) method to estimate standard errors.

We then examined the proportions of participants taking the most common PIM classes using logistic regression analysis, adjusted for all variables described above as they could affect medication use. Models examining metformin and sulfonylurea use were limited to those with self-reported prediabetes or diabetes. Regression results were used to estimate the adjusted proportions and 95\% CIs of PIM use, stratified by CKD stage and awareness [23]. Wald tests compared the adjusted risk difference for taking PIMs between CKD-aware and CKDunaware, within a given stratum of kidney function measured by eGFR; full details of the analysis are available in eMethods. Following best practice suggestions from the American Statistical Association [24], we report findings regardless of $p$ value. To provide context on the strength of the evidence supporting these findings, we used the Holm sequentially rejective multiple comparisons procedure [25] to control the family-wise error rate at 0.05 in the context of comparisons of five outcomes across three disease stages. We provide $p$ values and $95 \%$ confidence intervals and indicate where a $p$ value meets the Holm criterion. 
Analyses were performed in Stata 15.1 (StataCorp, College Station, TX) and SAS 9.4 (SAS Institute Inc., Cary, NC).

\section{RESULTS}

\section{Prevalence of CKD and CKD Awareness}

Prevalence of moderate/advanced CKD in the study population was $7.1 \%$ : $4.9 \%$ with stage $3 a, 1.6 \%$ with stage $3 b$, and $0.6 \%$ with stages $4-5 \mathrm{CKD}$, representing approximately $15.6,5.3$, and 2.0 million American adults, respectively. CKD awareness was poor, but improved with worsening kidney function: $91.6 \%$ of people with stage $3 \mathrm{a}, 73.1 \%$ with stage $3 \mathrm{~b}$, and $38.6 \%$ with stages $4-5$ CKD were unaware of their disease. Demographic and clinical characteristics of the study population, stratified by CKD stage and awareness, are presented in Table 1. People without CKD were substantially younger, with higher educational attainment, and with fewer comorbidities than those with CKD. Within each CKD stage, younger people, men, black or Hispanic people, and people with diabetes were more often CKD-aware.

\section{Prevalence of Potentially Inappropriate Medication Use}

Crude rates of PIM use were high among people with CKD across all CKD stages and awareness categories, ranging from 53.9\% (CKD-unaware stage $3 \mathrm{a}$ and CKD-aware stages 4-5) to $68.7 \%$ (CKD-aware, stage $3 \mathrm{~b}$ ) (Table 2). By comparison, these medications were used by $24.0 \%$ of people with a normal eGFR, in whom they are not considered inappropriate. The median and total numbers of PIMs taken by patients in different CKD stage and awareness categories are shown in Figure 1. Overall, the fewest PIMs were taken by CKD-unaware patients with stage $3 \mathrm{a} C K D$ and $C K D$-aware patients with stages 4-5 CKD (Fig. 1a), but many took multiple PIMs (Fig. 1b). PPIs, opioids, metformin, sulfonylureas, and NSAIDs were all commonly used, though there were no CKD-aware patients with stages 4-5 CKD taking metformin, bisphosphonates, or non-warfarin anticoagulants (Table 2).

The adjusted proportions of PIM use among people with CKD, overall and individually for the most common PIM classes, are shown in Figure 2 and complete regression results are available in the Supplementary Materials (eTables 2-7). There was no association between CKD stage or awareness category and odds of PIM use overall (eTable 2) or with use of opioids (eTable 4), sulfonylureas (eTable 6), or PPIs (eTable 7). While NSAID use was not associated with CKD stage, patients who were CKD-unaware were 6.2-times more likely to take NSAIDs than those who were CKD-unaware (eTable 3). People with prediabetes/diabetes and stages $3 \mathrm{~b}$ and 4-5 CKD were significantly less likely to take metformin than patients with stage $3 \mathrm{a}$ CKD, without clear impact of CKD awareness (eTable 5).
Among people with stage $3 \mathrm{a} \mathrm{CKD}$, the adjusted probability of NSAID use was $10.7 \%$ (95\% CI, 7.6 to 13.8) among the CKD-unaware compared with $2.2 \%$ (95\% CI, -0.3 to 4.7 ) of the CKD-aware (risk ratio 4.8; $p<0.001$ ). This was the only difference to meet Holm criterion for controlling the familywise error rate at 0.05 . Among people with stage $3 \mathrm{~b}$ CKD, probability of NSAIDs was 7.0\% (95\% CI, 2.6-11.4) among the CKD-unaware compared with 5.4\% (95\% CI, 0.3-10.5) among the CKD-aware (risk ratio $1.3 ; p=0.65$ ). Finally, among people with stages $4-5 \mathrm{CKD}$, probability of NSAIDs use was $16.5 \%$ (95\% CI, 4.0 to 29.0) among the CKDunaware compared with $0.8 \%$ (95\% CI, -0.9 to 2.5$)$ among the CKD-aware (risk ratio $19.6 ; p=0.01$ ).

The adjusted probability of metformin use was 3.3 times higher among people with prediabetes/diabetes and stage $3 \mathrm{~b}$ CKD who were CKD-unaware than unaware: $26.5 \%$ (95\% CI, $17.4-35.7)$ vs. $8.1 \%$ (95\% CI, $0.3-15.9)(p=0.003)$. In addition, $20.8 \%$ (95\% CI, 1.8-39.8) of CKD-unaware people with stages 4-5 CKD were estimated to take metformin; adjusted probability of metformin use among CKD-aware people with stages 4-5 CKD could not be calculated as no NHANES participants in this group reported metformin use. Sulfonylureas was predicted to be used by $24.1 \%$ (95\% CI, 17.6-30.7) and $17.0 \%$ (5.4-28.7) of people with stage $3 \mathrm{a}$ CKD when CKD-unaware and CKD-aware, respectively (risk ratio $1.4 ; p=0.30) ; 42.5 \%(29.2-55.8)$ and $34.8 \%(18.9-50.7)$ of people with stage $3 \mathrm{~b}$ CKD (risk ratio $1.2 ; p=0.53$ ); and $29.6 \%$ (8.4-50.7) and $15.3 \%$ (4.2-26.3) of patients with stages 4-5 CKD (risk ratio 1.9; $p=0.30$ ). Adjusted rates of PPI use ranged from $17.8 \%$ among CKD-unaware people with stages 4-5 CKD to $29.7 \%$ among CKD-aware patients with stage 3a CKD. Finally, adjusted rates of opioid use appeared higher among people with stage $3 \mathrm{a} C K D$ who were $\mathrm{CKD}$-aware than those who were unaware (23.0\% versus $9.2 \%$ ), were nearly identical across awareness in stage $3 \mathrm{~b}$ patients $(12.2 \%$ versus $12.0 \%$ ), and appeared lower among the CKD-aware with stages 4-5 CKD (11.2\% versus $19.0 \%)$, though these differences were not statistically significant: risk ratio with stage $3 \mathrm{a}$ was $0.4(p=0.15)$; stage $3 \mathrm{~b}$ was $0.99(p=0.98)$; and stage $4-5$ was $1.7(p=0.11)$.

\section{DISCUSSION}

Between 2011 and 2016, 7.1\% Americans were living with moderate or advanced CKD (eGFR $<60)$, though individual awareness of kidney disease was suboptimal, ranging from $8.4 \%$ with stage $3 \mathrm{a}$ CKD to $61.4 \%$ with stages $4-5 \mathrm{CKD}$. Use of medications known to be nephrotoxic, renally eliminated, or otherwise potentially harmful in the setting of CKD was common. After adjusting for factors potentially affecting PIM use (e.g., age, sex, race/ethnicity, comorbidities, insurance status), the proportion of people taking PIMs exceeded 50\% across all levels of kidney disease and CKD awareness. The only exception that met statistical significance criteria after 


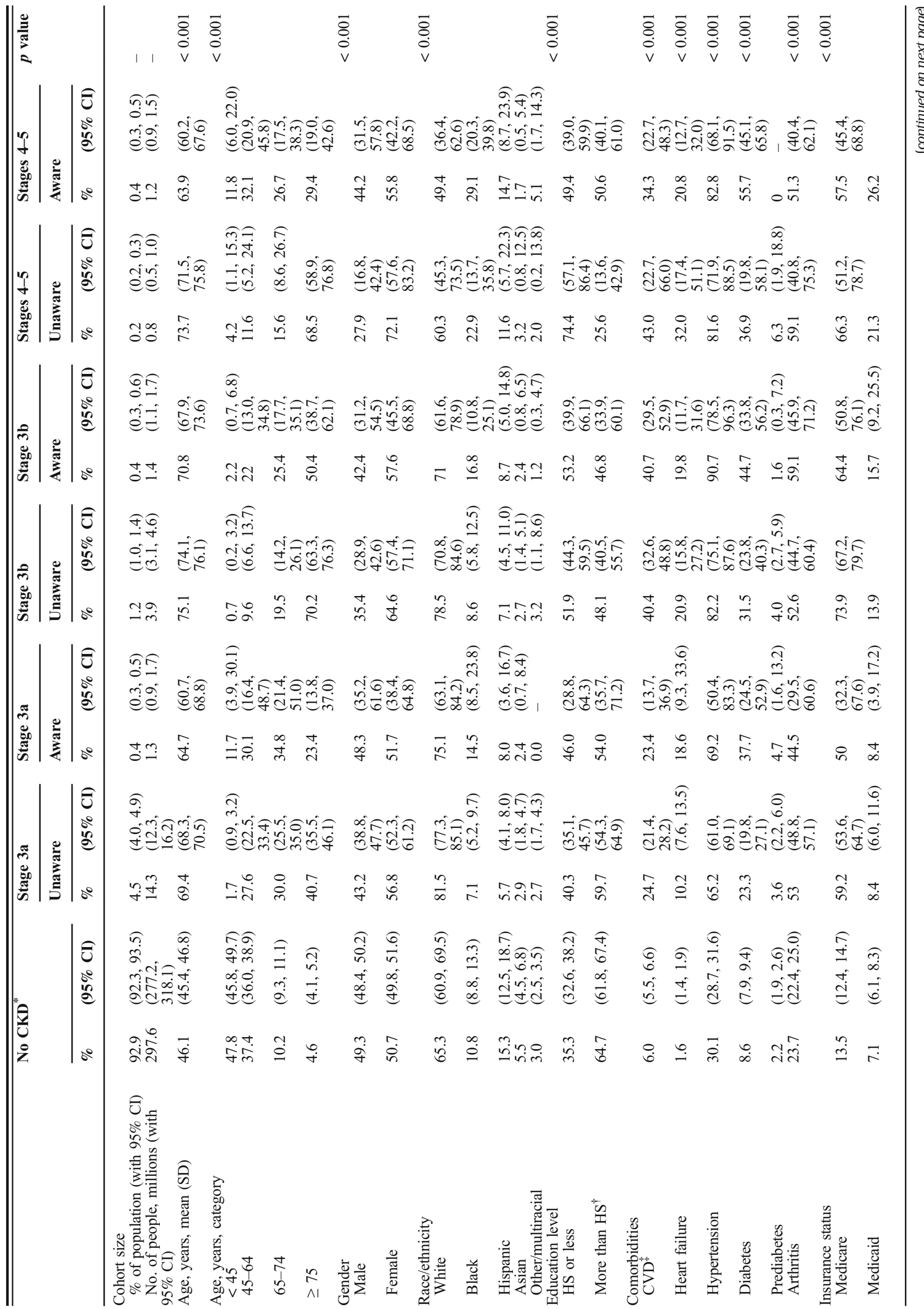


correction for multiple comparisons was NSAIDs, which were used nearly 5 times more frequently by people with stage 3 a CKD who were not aware of their disease. Other large observed differences, which were not robust to correction for multiple comparisons, were for NSAIDs with stages 4-5 CKD (20-fold higher rate when CKD-unaware), metformin with stage $3 \mathrm{~b}$ CKD (3-fold higher), and metformin with stages 45 CKD (used by $20.8 \%$ of CKD-unaware and none CKDaware). These findings suggest that a large number of people with CKD may be at risk for poor health outcomes due to high prevalence of PIM use, which may be impacted by gaps in CKD awareness.

Our study builds upon earlier work examining medication use in the context of CKD [26-30], though there was no data on impact of CKD awareness and no large-scale evaluation of multiple PIM classes in the general U.S. population had been performed to date. Our findings were consistent with the high rates of PIM use demonstrated by studies of older adults in the Veterans Health Administration (examining diabetes drugs) [29], privately insured beneficiaries (direct oral anticoagulants) [30], limited-income Medicare beneficiaries in two U.S. states (NSAIDs) [27], and several medical centers (NSAIDs) [26]. While some PIM use may represent carefully considered clinical reasoning with a patient-centered approach, particularly if no therapeutic alternative is available (e.g., allopurinol for gout), other cases may reflect a missed opportunity to select viable safer therapeutic alternatives (e.g., H2-receptor blocker instead of PPI for dyspepsia) or implement advanced monitoring. Moreover, our study may underestimate the prevalence of PIM use, as we focused on a narrow subset of PIMs specified by KDIGO Clinical Practice Guidelines [12], prescribing recommendations [18], and published literature [16, 19-21] and there are likely others associated with adverse health outcomes particularly among people with advanced CKD, due to potential for accumulation and/or toxicity.

High rate of PPI use among people with CKD (over 20\% across CKD stage and awareness categories) was not surprising. PPIs do not require dose adjustment and there is no specific eGFR threshold for caution with PPIs use. Nevertheless, there is emerging literature that PPIs are associated with increased risk of CKD incidence [31-36] and progression [32, $34,37]$ and patients with CKD may want to avoid or minimize PPI use particularly as there are other potentially viable alternatives for treating dyspepsia, gastroesophageal reflux disease, and peptic ulcer disease (i.e., H2 receptor blockers) [36].

Opioids should be used with caution in any circumstance, but particularly in the context of kidney disease [38]. Pain management in patients with CKD is challenging, as patients may rely on opioid anelgisia as their sole available therapeutic option due to contraindication of NSAIDs if acetaminophen and neuromodulatory medications are insufficient or similarly contraindicated. Nevertheless, reduced opioid clearance in impaired kidney function (even with stage 3a CKD) may lead to excessive sedation, myoclonus, seizures, suppressed 


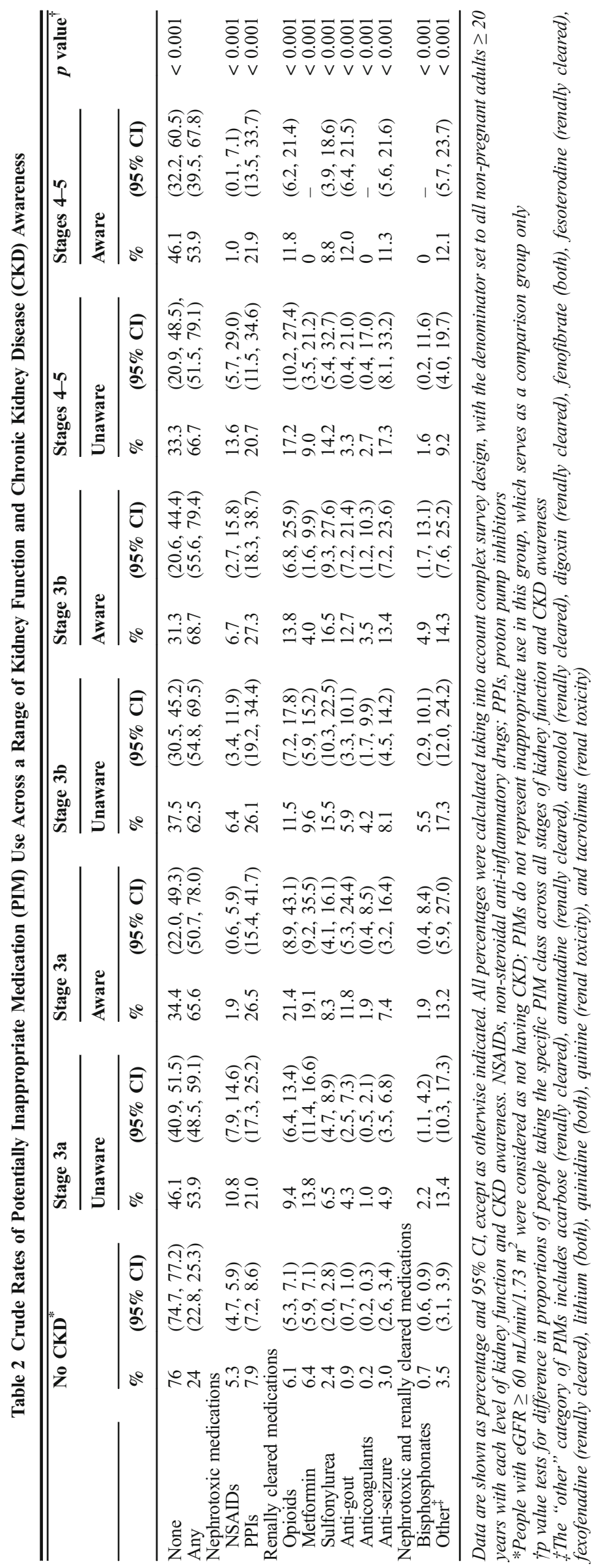



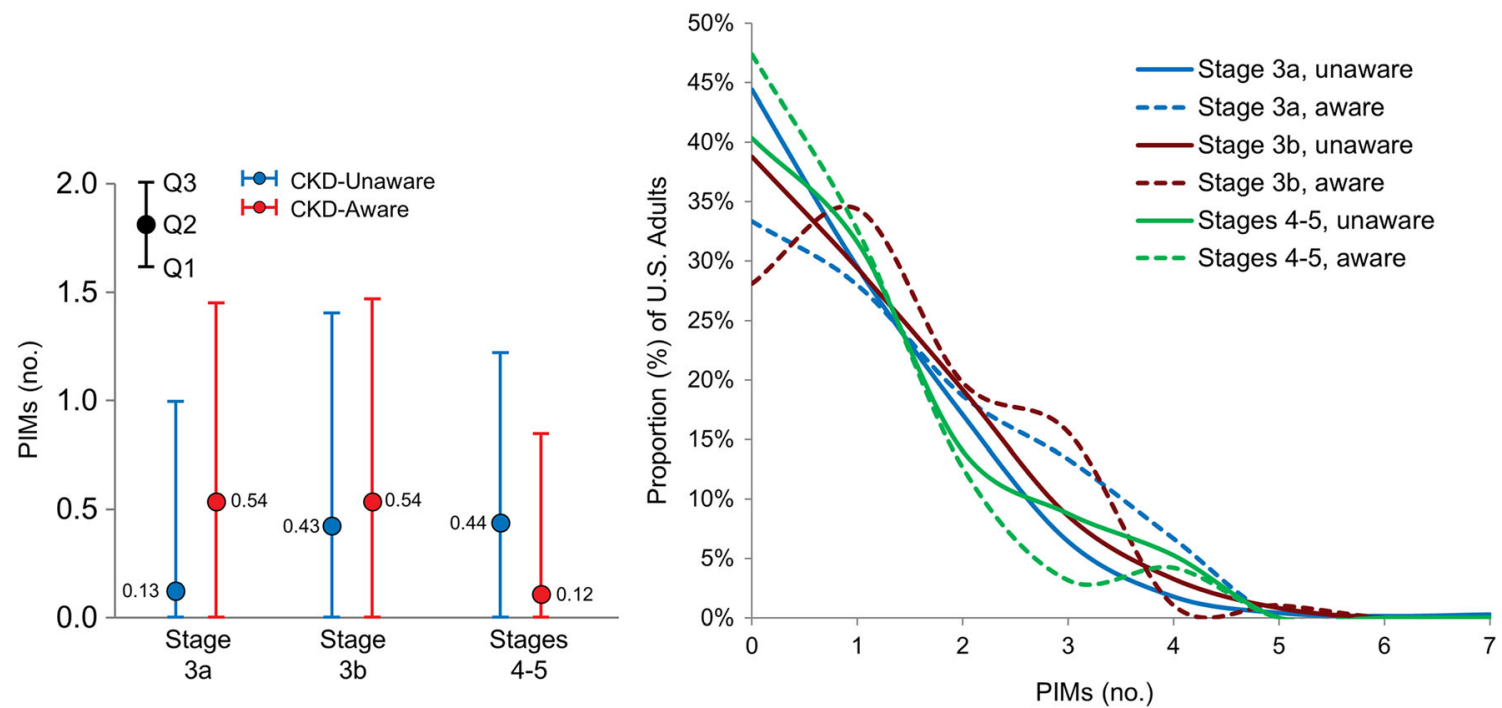

Figure 1 Potentially inappropriate medication use by people with chronic kidney disease. a Crude median (Q2) and interquartile range (Q1, Q3) numbers of potentially inappropriate medications used by U.S. adults with chronic kidney disease (CKD), stratified by CKD stage and awareness category. b Distribution of the total number of potentially inappropriate medications used by U.S. adults with chronic kidney disease, stratified by CKD stage and awareness category.

respiratory drive, and overdose [39]. This is particularly important as these symptoms may be misinterpreted as uremia rather than opioid toxicity/overdose. People with CKD and chronic pain who require opioids would benefit from using lowest effective doses, for the shortest duration of therapy, and with close monitoring; none of these factors could be assessed in our cross-sectional study design.

Diabetes is a leading cause of CKD [12] and over 25\% of people with diabetes also have kidney disease [40]. Metformin, while recommended as the first-line medication for the management of type 2 diabetes [41], was considered contraindicated with elevated creatinine/reduced eGFR throughout the study period, though in 2016 the U.S. Food and Drug Administration expanded its labeled use to those with eGFR $\geq$ 30 [42]. Metformin remains contraindicated with stages 4-5 CKD, yet an estimated $20.8 \%$ of CKD-unaware patients with prediabetes/diabetes and stages 4-5 CKD reported metformin use; importantly, none of the CKD-aware patients did. Metformin was also used less frequently by CKD-aware patients with stage $3 \mathrm{~b}$ CKD $(8.1 \%$ vs. $26.5 \%)$, consistent with current prescribing recommendations. These differences in metformin use in the presence of CKD awareness are clinically informative, even if not statistically significant after conservative adjustment for multiple comparisons. Sulfonylureas are also potentially inappropriate, as they increase the risk of hypoglycemia in the context of CKD $[41,43]$, though are not explicitly contraindicated. Sulfonylureas were often used by people of all CKD stages, with no significant association with CKD awareness (though point estimates for sulfonylurea use were consistently higher among the CKD-unaware).

We found clear evidence of an effect of CKD awareness on NSAID use, whose nephrotoxicity is well established [12, 44, 45]. The probability of NSAID use was lower among people with stages $3 \mathrm{a}$ and $4-5 \mathrm{CKD}$ only when they were CKD- aware. The adjusted proportions of people with stage $3 \mathrm{a}$ CKD taking NSAIDs were $10.7 \%$ vs. $2.2 \%$ for CKDunaware vs. CKD-aware, and for people with stages 4-5 CKD, $16.5 \%$ vs. $0.8 \%$. Clinicians and patients may be more aware of kidney-related considerations for medications like NSAIDs and metformin than others. Finally, the effect of CKD awareness in particular may be strongest for NSAIDs because they are typically obtained over-the-counter, and thus, the onus of avoidance falls on patients rather than clinicians. Although some PPIs are also available over-the-counter, they are more likely to be obtained upon a clinician's recommendation than NSAIDs, potentially reducing the patient's agency for their avoidance. These findings reinforce the potential benefit of educating people with CKD about their kidney disease and its implications on safe use of prescription and over-the-counter medications.

While high rates of PIM use among people with CKD are concerning and call for improved medication management in this high-risk population, our findings need to be considered in light of their limitations. NHANES does not fully capture each participant's comorbidity burden and patients with CKD, particularly higher stages of CKD, are more likely to have comorbidities that require the examined medications despite their potential for adverse effects in this population. We also could not assess the clinical contexts in which these medications were prescribed or the availability of and access to potentially safer alternatives. These results should therefore not preclude patients from receiving clinically necessary medications when dosed safely and under close monitoring. In addition, this is a cross-sectional study and diagnosing CKD based on a single creatinine and eGFR may incompletely reflect the participants' baseline kidney function. We captured medications used at the time of the NHANES interview, with no knowledge about duration of treatment. Medications such 


\begin{tabular}{|c|c|c|c|c|}
\hline CKD Stage $3 a$ & & $95 \% \mathrm{Cl}$ & & $\mathbf{P}$ \\
\hline \multirow[t]{2}{*}{ Any PIM } & Unaware & $54.1(49.3,58.9)$ & \multirow{2}{*}{$\stackrel{\text { 어 }}{10}$} & 0.23 \\
\hline & Aware & $64.4(48.1,80.6)$ & & \\
\hline \multirow[t]{2}{*}{ NSAIDs } & Unaware & $10.7(7.6,13.8)$ & \multirow{2}{*}{ 어 } & $<0.001^{\dagger}$ \\
\hline & Aware & $2.2(-0.3,4.7)$ & & \\
\hline \multirow[t]{2}{*}{ Opioids } & Unaware & $9.2(6.0,12.5)$ & \multirow{2}{*}{$\stackrel{101}{1}$} & 0.15 \\
\hline & Aware & $23.0(5.1,41.0)$ & & \\
\hline \multirow[t]{2}{*}{ Metformin* } & Unaware & $48.1(41.3,54.9)$ & \multirow{2}{*}{$10-1$} & 0.71 \\
\hline & Aware & $43.2(20.0,66.3)$ & & \\
\hline \multirow[t]{2}{*}{ Sulfonylureas* } & Unaware & $24.1(17.6,30.7)$ & \multirow{2}{*}{-OH } & 0.30 \\
\hline & Aware & $17.0(5.4,28.7)$ & & \\
\hline \multirow[t]{2}{*}{ PPIs } & Unaware & $20.8(16.9,24.6)$ & \multirow{2}{*}{ 어 } & 0.23 \\
\hline & Aware & $29.7(15.6,43.7)$ & & \\
\hline
\end{tabular}

\begin{tabular}{|c|c|c|c|c|}
\hline CKD Stage $3 b$ & & $95 \% \mathrm{Cl}$ & & $\mathbf{P}$ \\
\hline \multirow[t]{2}{*}{ Any PIM } & Unaware & $63.4(57.0,69.9)$ & \multirow{2}{*}{${ }_{1}^{\mathrm{HO}-1}$} & 0.69 \\
\hline & Aware & $66.2(53.5,78.8)$ & & \\
\hline \multirow[t]{2}{*}{ NSAIDs } & Unaware & $7.0(2.6,11.4)$ & \multirow{2}{*}{ 어 } & 0.65 \\
\hline & Aware & $5.4(0.3,10.5)$ & & \\
\hline \multirow[t]{2}{*}{ Opioids } & Unaware & $12.0(6.4,17.6)$ & \multirow{2}{*}{$\stackrel{\mathrm{HOH}}{\mathrm{HO}}$} & 0.98 \\
\hline & Aware & $12.2(4.1,20.2)$ & & \\
\hline \multirow[t]{2}{*}{ Metformin* } & Unaware & $26.5(17.4,35.7)$ & \multirow{2}{*}{${ }_{-1}^{-0-1}$} & 0.003 \\
\hline & Aware & $8.1(0.3,15.9)$ & & \\
\hline \multirow[t]{2}{*}{ Sulfonylureas* } & Unaware & $42.5(29.2,55.8)$ & \multirow{2}{*}{1} & 0.53 \\
\hline & Aware & $34.8(18.9,50.7)$ & & \\
\hline \multirow[t]{2}{*}{ PPIs } & Unaware & $25.9(18.7,33.0)$ & \multirow{2}{*}{$\stackrel{10-1}{10-1}$} & \multirow[t]{2}{*}{0.70} \\
\hline & Aware & $28.0(17.5,38.4)$ & & \\
\hline
\end{tabular}

\begin{tabular}{|c|c|c|c|c|}
\hline CKD Stages 4-5 & & $95 \% \mathrm{Cl}$ & & $P$ \\
\hline \multirow[t]{2}{*}{ Any PIM } & Unaware & $66.6(55.5,77.8)$ & $10-1$ & 0.14 \\
\hline & Aware & $53.9(40.0,67.8)$ & $10-1$ & \\
\hline \multirow[t]{2}{*}{ NSAIDs } & Unaware & $16.5(4.0,29.0)$ & $10-1$ & 0.02 \\
\hline & Aware & $0.8(-0.9,2.5)$ & & \\
\hline \multirow[t]{2}{*}{ Opioids } & Unaware & $19.0(11.0,27.1)$ & $10-1$ & 0.11 \\
\hline & Aware & $11.2(5.4,17.0)$ & $\mathrm{HOH}$ & \\
\hline \multirow[t]{2}{*}{ Metformin ${ }^{*}$} & Unaware & $20.8(1.8,39.8)$ & $1-1$ & $-\ldots$ \\
\hline & Aware & $0.0^{\ddagger}$ & & \\
\hline \multirow[t]{2}{*}{ Sulfonylureas* } & Unaware & $29.6(8.4,50.7)$ & 10 & 0.30 \\
\hline & Aware & $15.3(4.2,26.3)$ & $10-1$ & \\
\hline \multirow[t]{2}{*}{ PPIs } & Unaware & $17.8(8.3,27.2)$ & $10-1$ & 0.31 \\
\hline & Aware & $24.3(14.2,34.3)$ & $10-1$ & \\
\hline
\end{tabular}

Figure 2 Adjusted proportions of people using potentially inappropriate medication by stage of chronic kidney disease and awareness of it.

Proportions were adjusted for age, gender, race/ethnicity, education level, comorbidities (cardiovascular disease, heart failure, diabetes, prediabetes, arthritis), and insurance status. "Proportions of people using glucose-lowering medications were calculated from only among those with self-reported prediabetes or diabetes. 'Difference between CKD-aware and CKD-unaware remained statistically significant after Holm's adjustment for multiple comparisons.

as NSAIDs, opioids, and PPIs may confer less risk with limited exposure, which could not be assessed. Nevertheless, all of the PIMs examined in this study are candidates for close monitoring, limited and cautious use, and potential deprescribing in the setting of CKD, particularly in its advanced stages.

In summary, people with CKD are frequently treated with PIMs that have been associated with adverse health outcomes in this population. CKD awareness was poor, particularly among people with stages $3 \mathrm{a}-\mathrm{b} C K D$, yet may reduce the probability of some PIM use, particularly NSAIDs and metformin. Our study reinforces the importance of engaging people with CKD in periodic medication review and educating them about their kidney disease and its implications on medication management. Strategies to minimize PIM prescribing to at-risk individuals, facilitate deprescribing, and enhance monitoring for essential medications should also be evaluated and implemented.

Corresponding Author: Rozalina G. McCoy, MD, MS; Division of Community Internal Medicine, Department of Medicine Mayo Clinic, First Street SW, Rochester, MN, USA (e-mail: mccoy.rozalina@mayo. $e d u$ ).

Authors' Contributions Dr. McCoy and Ms. Kurani are the guarantors of this work and, as such, had full access to all the data in the study and take responsibility for the integrity of the data and the 
accuracy of the data analysis. They affirm that the manuscript is an honest, accurate, and transparent account of the study being reported; that no important aspects of the study have been omitted; and that any discrepancies from the study as planned have been explained. Dr. McCoy designed the study, interpreted the data, and drafted the manuscript. Ms. Kurani and Dr. Jeffery analyzed the data and reviewed/edited the manuscript. Dr. Thorsteinsdottir, Dr. Hickson, Ms. Barreto, Mr. Haag, Ms. Giblon, and Dr. Shah contributed to study design, data interpretation, and reviewed/edited the manuscript.

Funding Information This work was supported by the Mayo Clinic Robert D. and Patricia E. Kern Center for the Science of Health Care Delivery, the National Institute of Diabetes and Digestive and Kidney Diseases of the National Institutes of Health (K23DK114497 [McCoy] and K23DK109134 [Hickson]), and the National Institute on Aging of the National Institutes of Health (K23AG051679 [Thorsteinsdottir]).

\section{Compliance with Ethical Standards:}

Conflict of Interest: The authors have no relevant conflicts of interest to declare. In the past 36 months, Dr. McCoy was also supported by the $A A R P{ }^{\circledR}$ Quality Measure Innovation Grant through a collaboration with OptumLabs ${ }^{\circledR}$ and the NQF Measure Incubator. Dr. Jeffery has received research support through Mayo Clinic from the National Heart, Lung and Blood Institute (R56HL130496 and R21HL140287), the Agency for Healthcare Research and Quality (RO1HSO25164), the American Cancer Society (131611-RSGI-17-154-01-CPHPS), the Food and Drug Administration-funded Yale-Mayo CERSI (UO1FD 05938), and the National Center for Advancing Translational Sciences (UL1TR 02377; U01TR 02743). In the past 36 months, Dr. Shah has received research support through Mayo Clinic from the Food and Drug Administration to establish Yale-Mayo Clinic Center for Excellence in Regulatory Science and Innovation (CERSI) program (U01FDO05938); the Centers of Medicare and Medicaid Innovation under the Transforming Clinical Practice Initiative (TCPI); the Agency for Healthcare Research and Quality (R01HSO25164; RO1HSO25402; R03HSO25517); the National Heart, Lung and Blood Institute of the National Institutes of Health (NIH) (R56HL130496; R01HL131535); the National Science Foundation; and the Patient Centered Outcomes Research Institute (PCORI) to develop a Clinical Data Research Network (LHSNet).

Disclaimer: The content is solely the responsibility of the authors and does not necessarily represent the official views of the National Institutes of Health.

\section{REFERENCES}

1. CDC 2017 Pages. Accessed at US Department of Health and Human Services, Centers for Disease Control and Prevention, National Center for Chronic Disease Prevention and Health Promotion, Division of Diabetes Translation at https://www.cdc.gov/kidneydisease/pdf/kidney_ factsheet.pdf on January 102019.

2. Tuot DS, Plantinga LC, Hsu C-y, Powe NR. Is awareness of chronic kidney disease associated with evidence-based guideline-concordant outcomes? American journal of nephrology. 2012;35(2):191-7.

3. Plantinga LC, Tuot DS, Powe NR. Awareness of chronic kidney disease among patients and providers. Advances in chronic kidney disease. 2010;17(3):225-36.

4. Plantinga LC, Boulware LE, Coresh J, Stevens LA, Miller ER, 3rd, Saran R, et al. Patient awareness of chronic kidney disease: trends and predictors. Arch Intern Med. 2008;168(20):2268-75.

5. CDC [Website]. 2014;Pages. Accessed at Centers for Disease Control and Prevention at https://nccd.cdc.gov/ckd/data.aspx on February 142019.

6. Foreman $\mathbf{K J}$, Marquez $\mathbf{N}$, Dolgert A, Fukutaki $\mathbf{K}$, Fullman $\mathbf{N}$, McGaughey M, et al. Forecasting life expectancy, years of life lost, and all-cause and cause-specific mortality for 250 causes of death: reference and alternative scenarios for 2016-40 for 195 countries and territories. Lancet. 2018;392(10159):2052-90.

7. Fraser SD, Roderick PJ, May CR, McIntyre N, McIntyre C, Fluck RJ, et al. The burden of comorbidity in people with chronic kidney disease stage 3: a cohort study. BMC Nephrol. 2015;16:193.
8. Stevens LA, Li S, Wang C, Huang C, Becker BN, Bomback AS, et al Prevalence of CKD and comorbid illness in elderly patients in the United States: results from the Kidney Early Evaluation Program (KEEP). Am J Kidney Dis. 2010;55(3 Suppl 2):S23-33.

9. Tonelli M, Wiebe N, Guthrie B, James MT, Guan H, Fortin M, et al Comorbidity as a driver of adverse outcomes in people with chronic kidney disease. Kidney Int. 2015;88(4):859-66.

10. Lea-Henry TN, Carland JE, Stocker SL, Sevastos J, Roberts DM. Clinical pharmacokinetics in kidney disease. Fundamental Principles. 2018;13(7):1085-95.

11. Hemmelgarn BR, Manns BJ, Lloyd A, James MT, Klarenbach S, Quinn $\mathbf{R R}$, et al. Relation between kidney function, proteinuria, and adverse outcomes. JAMA. 2010;303(5):423-9.

12. KDIGO. KDIGO 2012 Clinical practice guideline for the evaluation and management of chronic kidney disease. Kidney International Supplements. 2013;3(1): 1-150

13. Adverse drug effects in relation to renal function: Jick H: Am J Med 62 514-517, 1977. The American Journal of Medicine. 1977;62(4):A97.

14. Brater DC. Drug dosing in patients with impaired renal function. Clin Pharmacol Ther. 2009;86(5):483-9.

15. Brownlee S, Garber J. Medication overload: America's other drug problem. In: Institute L, ed. Brookline, MA: Lown Institute; 2017.

16. Whittaker CF, Miklich MA, Patel RS, Fink JC. Medication safety principles and practice in CKD. Clinical Journal of the American Society of Nephrology. 2018;13(11):1738-46

17. Levey AS, Stevens LA, Schmid CH, Zhang YL, Castro AF, 3rd, Feldman HI, et al. A new equation to estimate glomerular filtration rate. Ann Intern Med. 2009;150(9):604-12.

18. Micromedex ${ }^{\circledR}$ (electronic version). Greenwood Village, Colorado, USA: IBM Watson Health; 2019.

19. McCoy AB, Waitman LR, Gadd CS, Danciu I, Smith JP, Lewis JB, et al A computerized provider order entry intervention for medication safety during acute kidney injury: a quality improvement report. American Journal of Kidney Diseases. 2010;56(5):832-41.

20. Moffett BS, Goldstein SL. Acute kidney injury and increasing nephrotoxic-medication exposure in noncritically-ill children. Clinical Journal of the American Society of Nephrology. 2011;6(4):856-63.

21. Ingrasciotta Y, Sultana J, Giorgianni F, Caputi AP, Arcoraci V, Tari DU, et al. The burden of nephrotoxic drug prescriptions in patients with chronic kidney disease: a retrospective population-based study in Southern Italy. PLoS One. 2014;9(2):e89072.

22. CDC;Pages. Accessed at U.S. Department of Health and Human Services at https://www.cdc.gov/nchs/tutorials/NHANES/NHANESAnalyses/ NHANES_Analyses_intro.htm on April 92019.

23. Norton EC, Dowd BE, Maciejewski ML. Marginal effects-quantifying the effect of changes in risk factors in logistic regression models. JAMA. 2019.

24. Wasserstein RL, Lazar NA. The ASA's statement on p-values: context, process, and purpose. The American Statistician. 2016;70(2):129-33.

25. Holm S. A simple sequentially rejective multiple test procedure. Scandinavian Journal of Statistics. 1979;6(2):65-70.

26. Zhan M, St. Peter WL, Doerfler RM, Woods CM, Blumenthal JB, Diamantidis CJ, et al. Patterns of NSAIDs use and their association with other analgesic use in CKD. Clinical Journal of the American Society of Nephrology. 2017;12(11):1778-86.

27. Winkelmayer WC, Waikar SS, Mogun H, Solomon DH. Nonselective and cyclooxygenase-2-selective NSAIDs and acute kidney injury. Am J Med. 2008;121(12):1092-8.

28. Farag A, Garg AX, Li L, Jain AK. Dosing errors in prescribed antibiotics for older persons with CKD: a retrospective time series analysis. Am J Kidney Dis. 2014;63(3):422-8.

29. Tseng CL, Soroka O, Maney M, Aron DC, Pogach LM. Assessing potential glycemic overtreatment in persons at hypoglycemic risk. JAMA Intern Med. 2014;174(2):259-68.

30. Yao X, Tangri N, Gersh BJ, Sangaralingham LR, Shah ND, Nath KA, et al. Renal outcomes in anticoagulated patients with atrial fibrillation. J Am Coll Cardiol. 2017;70(21):2621-32.

31. Lazarus B, Chen Y, Wilson FP, Sang Y, Chang AR, Coresh J, et al Proton pump inhibitor use and the risk of chronic kidney disease. JAMA Intern Med. 2016;176(2):238-46.

32. Xie Y, Bowe B, Li T, Xian H, Balasubramanian S, Al-Aly Z. Proton pump inhibitors and risk of incident CKD and progression to ESRD. J Am Soc Nephrol. 2016;27(10):3153-63.

33. Arora P, Gupta A, Golzy M, Patel N, Carter RL, Jalal K, et al. Proton pump inhibitors are associated with increased risk of development of chronic kidney disease. BMC Nephrol. 2016;17(1):112 
34. Xie Y, Bowe B, Li T, Xian H, Yan Y, Al-Aly Z. Long-term kidney outcomes among users of proton pump inhibitors without intervening acute kidney injury. Kidney Int. 2017;91(6): 1482-94.

35. Rodriguez-Poncelas A, Barcelo MA, Saez M, Coll-de-Tuero G. Duration and dosing of proton pump inhibitors associated with high incidence of chronic kidney disease in population-based cohort. PLoS One. 2018;13(10):e0204231.

36. Giu T, Zhou J, Zhang C. Acid-suppressive drugs and risk of kidney disease: a systematic review and meta-analysis. J Gastroenterol Hepatol. 2018

37. Klatte DCF, Gasparini A, Xu H, de Deco P, Trevisan M, Johansson ALV, et al. Association between proton pump inhibitor use and risk of progression of chronic kidney disease. Gastroenterology. 2017; 153(3):702-10.

38. Davison SN. Clinical pharmacology considerations in pain management in patients with advanced kidney failure. Clinical Journal of the American Society of Nephrology. 2019:https://doi.org/10.2215/CJN.05180418.

39. Dean M. Opioids in renal failure and dialysis patients. J Pain Symptom Manage. 2004;28(5):497-504.

40. Afkarian M, Zelnick LR, Hall YN, Heagerty PJ, Tuttle $K$, Weiss NS et al. Clinical manifestations of kidney disease among US adults with diabetes, 1988-2014. JAMA. 2016;316(6):602-10.
41. ADA. American Diabetes Association Standards of Medical Care in Diabetes-2019. Section 9. Pharmacologic Approaches to Glycemic Treatment. Diabetes Care. 2019;42(Supplement 1):S90-S102.

42. FDA 2016;Pages. Accessed at U.S. Food and Drug Administration at https://www.fda.gov/Drugs/DrugSafety/ucm493244.htm on February 212019

43. Silbert R, Salcido-Montenegro A, Rodriguez-Gutierrez R, Katabi A, McCoy RG. Hypoglycemia among patients with type 2 diabetes: epidemiology, risk factors, and prevention strategies. Curr Diab Rep. 2018;18(8):53.

44. Williams AW, Dwyer AC, Eddy AA, Fink JC, Jaber BL, Linas SL, et al. Critical and honest conversations: the evidence behind the "Choosing Wisely" campaign recommendations by the American Society of Nephrology. Clin J Am Soc Nephrol. 2012;7(10):1664-72.

45. Schneider V, Levesque LE, Zhang B, Hutchinson T, Brophy JM. Association of selective and conventional nonsteroidal antiinflammatory drugs with acute renal failure: a population-based, nested case-control analysis. Am J Epidemiol. 2006;164(9):881-9.

Publisher's Note Springer Nature remains neutral with regard to jurisdictional claims in published maps and institutional affiliations. 